Epidemiology and Infection

cambridge.org/hyg

\section{Original Paper}

Cite this article: Kumar A, Gittens-St Hilaire M, Nielsen AL (2018). Long-term epidemiological dynamics of dengue in Barbados - one of the English-speaking Caribbean countries. Epidemiology and Infection 146, 1048-1055. https://doi.org/10.1017/S0950268818000900

Received: 6 October 2017

Revised: 21 March 2018

Accepted: 22 March 2018

First published online: 15 April 2018

\section{Key words:}

Barbados; Caribbean region; climatic factors; dengue epidemiology; incidence trends

\section{Author for correspondence:}

A. Kumar, E-mail: alok.kumar@cavehill.uwi. edu; alokkumar.uwichill@gmail.com

\title{
Long-term epidemiological dynamics of dengue in Barbados - one of the English-speaking Caribbean countries
}

\author{
A. Kumar ${ }^{1,2}$, M. Gittens-St Hilaire ${ }^{1,3}$ and A. L. Nielsen ${ }^{1}$
}

\footnotetext{
${ }^{1}$ The University of the West Indies (Cave Hill), Bridgetown, Barbados; ${ }^{2}$ The Queen Elizabeth Hospital, Bridgetown, Barbados and ${ }^{3}$ Public Health Laboratory Dengue \& Leptospira Division, Government of Barbados, Bridgetown, Barbados
}

\begin{abstract}
Using the dengue surveillance program, we prospectively collected data on all the suspected and confirmed cases of dengue in Barbados from 2006 to 2015. Data were analysed for demographic, seasonal and temporal dynamics of this disease in this country. The overall mean annual incidence rate of suspected and confirmed dengue over the study period was $0.49 \%$ (range $0.15 \%-0.99 \%$ ) and $0.16 \%$ (range $0.05 \%-0.48 \%$ ), respectively. There was a significant correlation between the mean monthly number of confirmed cases, the mean monthly rainfall and the mean monthly relative humidity percentage. Dengue in this population is predominantly an infection affecting children and young adults. The median age of the patients with both, suspected and confirmed dengue was 25 years and the highest proportion of cases was seen in the age group 0-15 years. The annual incidence rates of both the suspected and the confirmed cases showed an upward trend during the study period and this upward trend was more pronounced among children.
\end{abstract}

\section{Introduction}

Over the past 15 years, the Americas have seen an unprecedented number of reported dengue cases with an alarmingly increasing trend [1-5]. Latin America and the Caribbean have reported the highest incidence of dengue worldwide with over two-thirds of all cases reported from this region during the 2000-2006 [1, 2]. In 2010 and in 2013, the English-speaking Caribbean was one of the worst affected regions in the Americas [3, 6, 7]. Dengue is reported to be hyper-endemic in most of the English-speaking Caribbean, including Barbados, with an epidemic occurring every 4-5 years during the period 1997 through 2009 [7-9]. All four serotypes of dengue have been circulating in the Caribbean and since 2001, all of the serotypes have been concurrently isolated in some of the countries, including Barbados [9, 10]. The long-term patterns of dengue incidence have been studied at numerous endemic sites in southeast Asia [11, 12]. There are some meta-analyses on the long-term incidence pattern from the Americas [8, 13-17]. Results from these studies highlight intra-annual (seasonal) and interannual (across multiple years) signatures in the transmission intensity [11-16], as well as shifts in the age of people with clinically apparent illness $[16,17]$. Conclusions from these studies are mixed, although, in aggregate, they highlight that dengue occurs across a diverse array of conditions and that the key drivers of transmission vary across those different conditions [11-17]. Continued and detailed documentation of these temporal dengue patterns in different endemic populations is useful for improving our understanding of the dynamics of dengue epidemics as well as DENV transmission and for testing the link of key variables like rainfall to components of the virus transmission cycle.

Barbados, one of the English-speaking Caribbean countries, is a commonwealth, independent nation and has a total population of 285000 (2016) including 54599 (22\%) under 15 children and a population density of $662.8 / \mathrm{sq} \mathrm{km}$. The majority $(90 \%)$ of the population are of African descent [18]. The Government of Barbados provides free health care to its citizens through polyclinics, which are the primary care centers and a single tertiary care hospital. People also use private healthcare facilities by paying out of pocket. Barbados has an Active Dengue Surveillance System (ADSS) in place since 2000. Under this system, all the cases of suspected dengue are tested for confirmation and the testing is provided free of charge to all patients attending both public and private healthcare facilities. There is a central dengue testing laboratory. Patients with suspected dengue are usually managed at the discretion of the attending physician along the WHO guidelines and blood samples for dengue confirmation are routinely collected from all suspected cases at the time of presentation or on a follow up visit around day 5 of illness, in case patients present during day 1 or 2 of their illness. Taking advantage of the unique health care system in this closed population, we used the ADSS to study the long-term epidemiologic and the seasonal dynamics of dengue in Barbados. 


\section{Material and methods}

\section{Study design and patient selection}

This is a prospective population-based study. The study period extends from 2006 to 2015. Cases of suspected Dengue were prospectively identified from the register at the central dengue laboratory. All suspected cases of dengue in adults and children from both, the private care settings and the public settings of the polyclinics and the hospital were included in this study. Cases of dengue among visitors to this island were excluded from this study. A preplanned analysis of relevant epidemiological data on all the suspected and confirmed cases of dengue and the local meteorological data were used to describe the dynamics of dengue transmission in this population. Local data were also compared with the available regional data obtained from the Pan American Health Organization (PAHO) for similarity and differences, if any, in the transmission dynamics between Barbados and other Caribbean countries which share similar climatic conditions.

\section{Patient data}

Demographic data such as the age, gender, date of onset of the illness and timing of the blood collection for dengue testing were prospectively abstracted from their laboratory requisition form. Any missing information with respect to the demography, date of onset or the timing of blood collection for any of the suspected cases were obtained from the patients case notes at the clinics where the patient was seen.

\section{Laboratory confirmation}

As per established protocol at the dengue laboratory, blood samples collected during the first 4 days of the illness are tested for the NS1 antigen along with IgM antibodies and blood samples collected after day 5 of illness are tested for the Dengue IgM antibodies. NS1 antigen testing is done using Platelia ${ }^{\mathrm{TM}}$ Dengue NS1 Ag-ELISA (Biorad Laboratories, Marnes-La-Coquette, France). Dengue IgM antibody-capture ELISAs (Focus Diagnostics, Cypress, CA, 90630 USA) were used for the IgM detection. The dengue test results for all the cases of suspected dengue were actively followed up. In those cases where the blood sample was negative and the blood sample was taken during the first 4 days of the illness then the patients' attending physician was advised to request a second blood sample from the patient to be taken during day $5-15$ of the illness for retesting.

\section{Local meteorological data}

Data on the annual rainfall, humidity and temperature for Barbados over the study period were obtained from the Meteorological department, Government of Barbados. Data on the local serotypes circulation, both the current and the past, were collected from our central dengue laboratory in Barbados.

\section{Regional data}

Additional data on the reported number and incidence of dengue for the Caribbean were collected from the PAHO website [19]. These data were used to compare the local dengue transmission pattern with those of the other Caribbean and Latin American countries with similar climatic conditions.

\section{Case definition and outcome measure}

As per the guidelines from the Ministry of Health, dengue is suspected in any person presenting with fever of duration more than $24 \mathrm{~h}$ with one or more signs and symptoms of dengue such as aches and pains, vomiting or nausea, rash, bleeding, abdominal pain, lethargy or hepatomegaly and where other causes of the fever are not obvious at the time of first presentation. A confirmed case of dengue was defined as one that had a positive IgM titer and/or positive NS1 (non-structural protein 1) antigen. The overall mean annual incidence rate was calculated by dividing the total number of cases over the study period by the population (based on 2010 census) and expressed as a percentage. The annual incidence rate was calculated by dividing the number of cases for the year by the population (based on 2010 census) and expressed as a percentage. The number and the incidence rate of both suspected and the confirmed dengue detected during this prospective surveillance study were compared with those reported from other Caribbean countries to the PAHO [19]. The years where the number of cases of dengue showed a peak were designated as epidemic years.

\section{Data management and statistical analysis}

Data were stored in a specially designed Microsoft $t^{\circledR}$ Access database and was analysed using SPSS $^{\circledast}$ statistical software package version 11 . Proportion and $95 \%$ confidence interval (CI) were calculated and results were corrected for continuity. Associations between categorical variables were assessed for statistical significance by $\chi^{2}$ test. A $P$ value of $\leqslant 0.05$ was considered statistically significant. All $P$ values were two-tailed.

\section{Ethics approval}

Necessary ethical approval for this study was obtained from the Institutional Review Board on Ethics in Research on human subjects at the University of the West Indies.

\section{Results}

Over the 10-year study period there were 13991 cases of febrile illnesses where dengue was suspected and a sample was sent for dengue confirmation. Over the same period, $4344(31.10 \%$ those tested) cases were confirmed as dengue. Diagnosis of dengue was confirmed based on a positive IgM serology in 3840 (88.4\%) cases, based on a positive NS1 antigen in $384(8.8 \%)$ cases and based on both NS1 and IgM positive results in 120 (2.8\%) cases. All 13991 cases had at least one sample tested for IgM antibody and 3960 (28.3\%) cases were positive. NS1 antigen test was done in 1448 cases and 504 (34.8\%) were positive.

The overall mean annual incidence rate of suspected and confirmed dengue over the study period was $0.49 \%$ (Range $0.15 \%$ $0.99 \%$ ) and $0.16 \%$ (Range $0.05 \%-0.48 \%$ ), respectively, compared with the reported pooled mean annual incidence of confirmed cases for the Caribbean at $0.058 \%(P<0.001)$, over the same period. Table 1 shows a comparison of the number and the incidence rate of the suspected and the confirmed dengue reported in this study with those reported from other countries in the Caribbean region to the PAHO. In Barbados, the number of both the suspected and the confirmed dengue peaked during the years 2007, 2010 and 2013 and were designated as the epidemic years. The reported number and the incidence of suspected 
Table 1. Dengue incidence and serotype circulation in Barbados compared with those reported from other Caribbean countries to Pan American Health Organization (PAHO) during 2006-2015

\begin{tabular}{|c|c|c|c|c|c|c|c|}
\hline \multirow[b]{2}{*}{ Years } & \multicolumn{2}{|c|}{ Hispanic Caribbean (data from PAHO) } & \multicolumn{2}{|c|}{$\begin{array}{l}\text { Non-Hispanic Caribbean (data from } \\
\text { PAHO) }\end{array}$} & \multicolumn{3}{|c|}{ Barbados } \\
\hline & $\begin{array}{l}\text { Suspected cases } \\
\text { (Incidence } \\
\text { rate-\%) }\end{array}$ & $\begin{array}{l}\text { Confirmed cases } \\
\text { (Incidence } \\
\text { rate- } \%)\end{array}$ & $\begin{array}{l}\text { Suspected cases } \\
\text { (Incidence } \\
\text { rate-\%) }\end{array}$ & $\begin{array}{l}\text { Confirmed cases } \\
\text { (Incidence } \\
\text { rate-\%) }\end{array}$ & $\begin{array}{l}\text { Suspected } \\
\text { cases } \\
\text { (Incidence } \\
\text { rate-\%) }\end{array}$ & $\begin{array}{l}\text { Confirmed } \\
\text { cases } \\
\text { (Incidence } \\
\text { Rate-\%) }\end{array}$ & $\begin{array}{l}\text { Serotype in } \\
\text { circulation }\end{array}$ \\
\hline 2006 & $9186(0.039)$ & $1187(0.005)$ & $23556(0.299)$ & $4289(0.054)$ & $419(0.148)$ & $153(0.054)$ & 3 \\
\hline 2007 & $20668(0.087)$ & $9180(0.039)$ & $10987(0.131)$ & No data & $1276(0.451)$ & $608(0.215)$ & 3 \\
\hline 2008 & $7717(0.033)$ & $2779(0.012)$ & $4681(0.054)$ & $1736(0.022)$ & $963(0.340)$ & $276(0.097)$ & 3 \\
\hline 2009 & $15013(0.063)$ & $7020(0.030)$ & $19673(0.247)$ & $7303(0.092)$ & $703(0.248)$ & $140(0.049)$ & 2,3 \\
\hline 2010 & $32817(0.138)$ & $16768(0.071)$ & $99829(1.258)$ & $13190(0.166)$ & $2000(0.707)$ & $575(0.203)$ & $1,2,3,4$ \\
\hline 2011 & $7993(0.034)$ & $2445(0.010)$ & $19442(0.246)$ & $8066(0.102)$ & $945(0.334)$ & $221(0.078)$ & $1,2,4$ \\
\hline 2012 & $22542(0.096)$ & $5652(0.024)$ & $17786(0.224)$ & $5475(0.069)$ & $1821(0.643)$ & $492(0.173)$ & $1,2,4$ \\
\hline 2013 & $36252(0.154)$ & $9637(0.041)$ & $51360(0.647)$ & $16820(0.213)$ & $2790(0.986)$ & $1350(0.477)$ & $1,2,4$ \\
\hline 2014 & $17767(0.071)$ & No data & $16639(0.087)$ & $5204(0.027)$ & $2529(0.894)$ & $407(0.144)$ & 1 \\
\hline 2015 & $20606(0.080)$ & No data & $8676(0.045)$ & $1918(0.010)$ & $545(0.193)$ & $127(0.045)$ & 1 \\
\hline
\end{tabular}

Note: numbers in bold are those for the epidemic years for Barbados.

and confirmed dengue reported to the PAHO from the other countries in the Caribbean also peaked in 2007, 2010 and 2013. However, the incidence rate of dengue in Barbados during these epidemic years was significantly higher than those of other Caribbean countries $(P<0.001)$. In Barbados, DENV3 was the only serotype in circulation during 2006-2008. The DENV2 appeared in 2009 when both DENV3 and DENV2 were in circulation. In 2010, DENV 1 was reintroduced along with the introduction of DENV4 and all four serotypes were simultaneously in circulation. Since 2011 up until the end of 2013, DENV1, DENV2, DENV4 were simultaneously in circulation. Since 2014 only DENV1 has been in circulation.

The annual number of suspected and confirmed cases along with the annual rainfall pattern in Barbados is shown in Figure 1. The annual total rainfall peaked during the years 2008, 2010 and 2013 and coincided with the peak number of cases in 2010 and 2013. The total annual rainfall troughed in

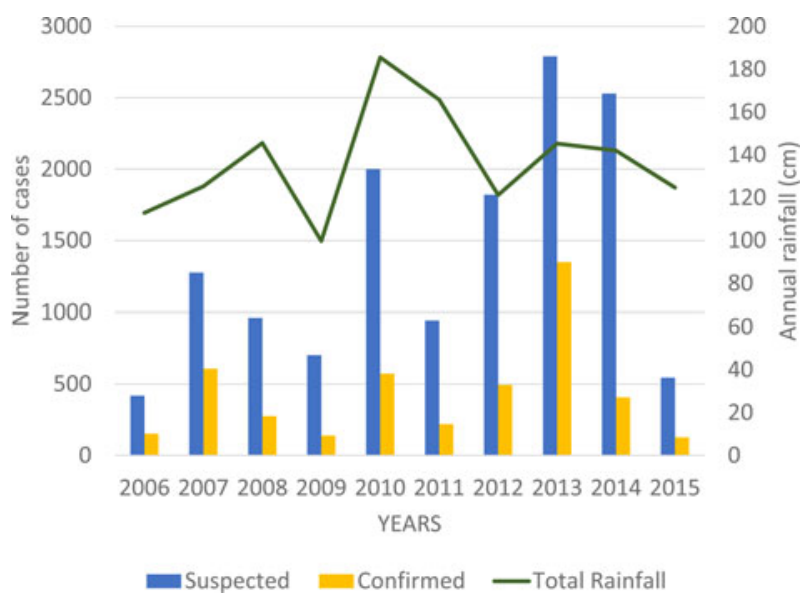

Fig. 1. Total annual rainfall and the number of dengue cases in Barbados, 2006-2015.
2009, 2012 and 2015 and coincided with the trough number of both the suspected and the confirmed cases during 2009 and 2015. However, no significant correlation was seen between the total annual rainfall and the total annual number of suspected $(P=0.2049)$ or confirmed cases $(P=0.4045)$. Figure 2 shows the mean monthly climatic data and the mean monthly number of cases during the 10-year study period. When the mean monthly climatic data were analysed with a monthly number of cases, there was a significant correlation between the mean monthly number of confirmed cases and the mean monthly rainfall $(P=0.0026)$ and with the mean monthly relative humidity $(P=0.0142)$. No significant correlation was found between the monthly cumulative number of confirmed cases and the minimum or maximum temperature.

The overall age and sex distribution of the suspected and confirmed cases are shown in Table 2. The median age of the patients with both, suspected and confirmed dengue was 25 years with the highest proportion of cases seen in the age group $0-15$ years. The difference in the proportion of suspected cases $(P<0.0001)$ and confirmed cases $(P<0.0001)$ in each age group category was statistically significant. Females had a significantly higher proportion of both the suspected $(P<0.0001)$ and confirmed cases $(P<$ $0.0001)$ compared with males. The difference in the proportion of suspected cases that was confirmed dengue in the various age groups was statistically not significant $(P=0.062)$. The proportion of suspected cases that were confirmed dengue during the epidemic years was at $41.53 \%$, whereas the corresponding figure during the non-epidemic years was at $24.94 \%$ and this difference was statistically significant (two-tailed $P=0.0165$ ).

The mean annual incidence rate of suspected cases among children $(<15$ years) and the adults were $0.75 \%$ and $0.43 \%$ respectively, and this difference was statistically significant $(P=$ 0.0026). The annual incidence rates of suspected and confirmed cases among children and adults (overall), among children and among adult were analysed and they all showed an upward trend during the 10-year study period. The annual incidence 


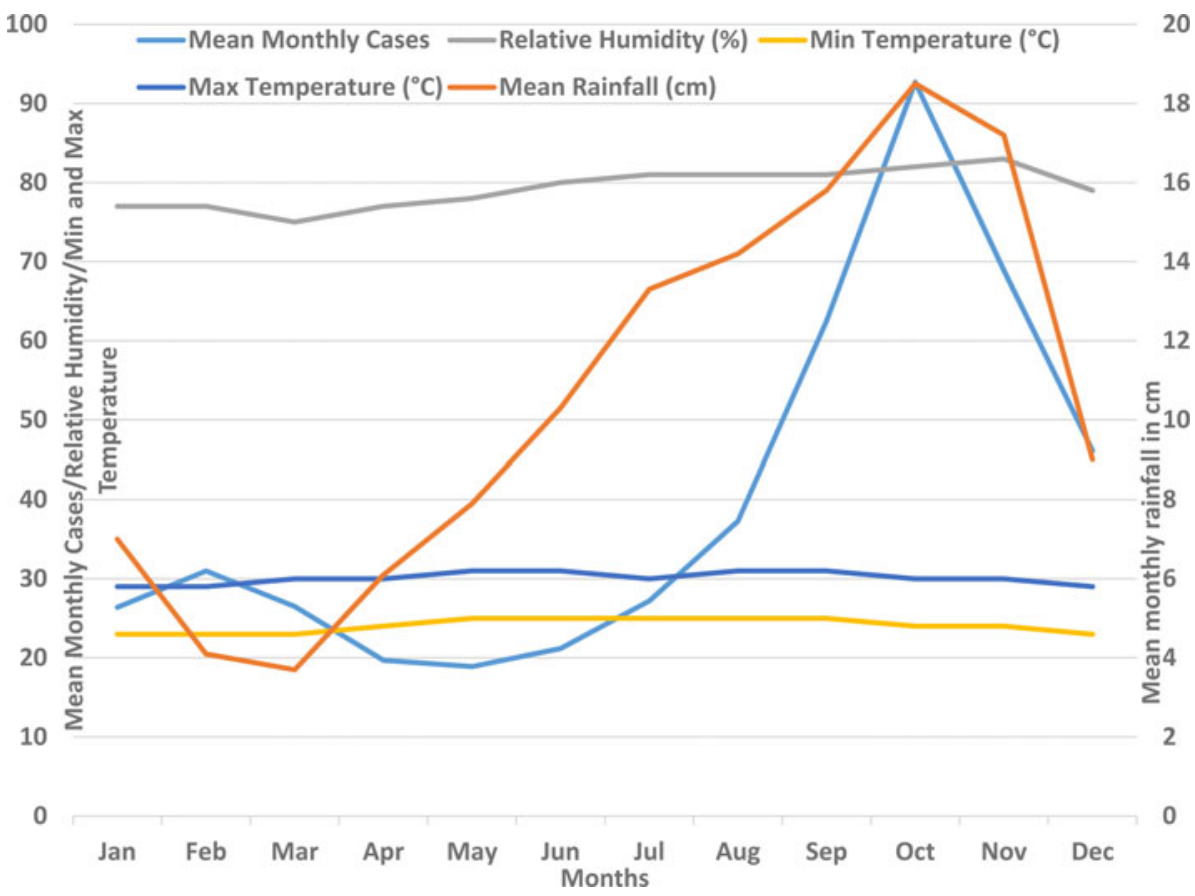

Fig. 2. Mean monthly rainfall and mean monthly number of confirmed dengue cases in Barbados, 2006-2015. rate of suspected cases of dengue is shown in Figure 3. During the study period, there was an increasing upward trend noted in the annual incidence rate of suspected dengue among children and this upward trend was statistically significant $\left(R^{2}=0.2424\right)$. An increasingly upward trend in the annual incidence rate of suspected dengue among adults was also noted, however, this was statistically not significant $\left(R^{2}=0.199\right)$. During the peak (epidemic) years 2007, 2010 and 2013 there was a consistent increase in the overall (children and adults) annual incidence rate of

Table 2. Demographic characterisation of the dengue epidemics in Barbados, 2006-2015

\begin{tabular}{|c|c|c|c|}
\hline Demography & $\begin{array}{c}\text { No. of } \\
\text { suspected cases } \\
\text { (\% of total } \\
\text { suspected) }\end{array}$ & $\begin{array}{c}\text { No. of } \\
\text { confirmed } \\
\text { cases ( } \% \text { of total } \\
\text { confirmed) }\end{array}$ & $\begin{array}{l}\text { Proportion of } \\
\text { laboratory } \\
\text { confirmation } \\
\text { among suspected } \\
\text { cases (\%) }\end{array}$ \\
\hline \multicolumn{4}{|l|}{ Age group } \\
\hline $0-14$ & $3896(27.85)$ & $1121(25.81)$ & 28.6 \\
\hline $15-29$ & 3631 (25.95) & 1119 (25.69) & 30.9 \\
\hline $30-44$ & 2678 (19.14) & 814 (18.73) & 30.4 \\
\hline $45-59$ & $1890(13.51)$ & 648 (14.92) & 34.3 \\
\hline $60-74$ & $861(6.16)$ & $288(6.64)$ & 33.5 \\
\hline $75-89$ & $396(2.83 \%)$ & $125(2.89)$ & 31.7 \\
\hline $90-104$ & $64(0.46)$ & $24(0.54)$ & 36.6 \\
\hline Not specified & $574(4.1)$ & $208(4.78)$ & 36.1 \\
\hline \multicolumn{4}{|l|}{ Gender } \\
\hline Female & 7491 (53.55) & $2367(53.47)$ & 39.63 \\
\hline Male & $6362(45.47)$ & $1945(45.64)$ & 38.27 \\
\hline Not specified & $138(0.98)$ & $32(0.89)$ & 30.93 \\
\hline
\end{tabular}

suspected dengue and this increase was highly significant $(P=$ 0.0002). During the trough years 2006, 2009, 2011 and 2015 change in the overall annual incidence rate of suspected dengue was inconsistent and varied within a narrow range $(0.15 \%$ and $0.33 \%)$. The difference in the annual incidence rates of suspected cases during the trough years was statistically not significant $(P=$ 0.0621 ). The mean annual incidence rate of confirmed dengue among children and adults was $0.23 \%$ and $0.13 \%$, respectively, and this difference was statistically significant $(P=0.0022)$. The pattern in the annual incidence rate of confirmed dengue in children and in adults during the study period and during the epidemic and non-epidemic years mirrored the pattern seen for the suspected cases (Fig. 4).

\section{Discussions}

Results from this longitudinal study reveal the dynamics of dengue epidemiology in Barbados. There are several long-term studies, mostly from south-east Asia on dengue transmission that have sought to define the dynamics of dengue epidemiology [12, 19-21]. However, more studies are required from the Caribbean to quantify the disease burden in different populations, explore the impact of DENV serotype-specific transmission on host-responses and dengue severity and measure the economic impact of dengue in this population. A long-term dengue transmission study is especially required from the English-speaking Caribbean, where there is a high intensity of transmission and paucity of such studies $[8,9,22]$. Findings from this long-term prospective study provide the epidemiologic data that is important for describing the dynamics of dengue epidemiology and for planning control measures [4, 23, 24]. The findings from this study are especially important in the context of the fact that Latin America and the Caribbean have been projected to be the region of the highest dengue transmission in the world with increasing frequency and intensity of dengue epidemics 


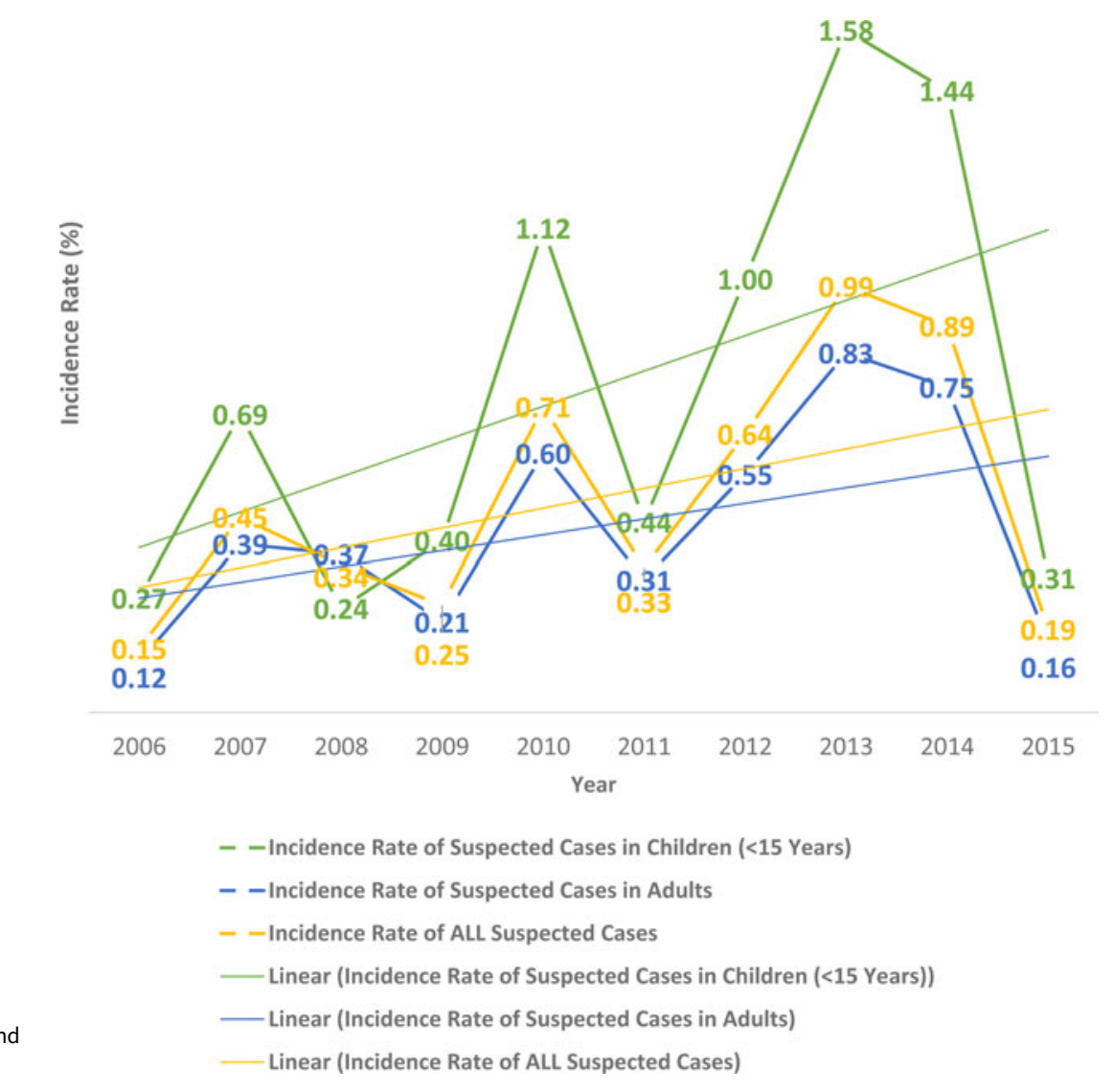

Fig. 3. Incidence rate of suspected dengue in children and adults, Barbados, 2006-2015.

over the past decade based on data that is limited in terms of quality, completeness and coverage $[1,3,6,25]$.

Dengue in Barbados occurs throughout the year with cases occurring each month in every year throughout the study period. Epidemics of dengue were seen in 2007, 2010 and 2013, all these 3 years also witnessed a pan-Caribbean epidemic (Table 1) and the years 2010 and 2013 witnessed a Pan-American epidemic [6]. The inter-epidemic period of 3 years seen in this country is shorter than the 3-5 years seen in our previous report over the period 2000-2009 [8]. Similar observations of shorter inter-epidemic durations have been made in some other recent studies from the Americas [26, 27]. The mean annual incidence of suspected dengue over the study period in this population was $0.49 \%$ (Range $0.14 \%-0.99 \%)$. This was significantly higher than the reported pooled mean annual incidence of suspected cases for the Americas (Latin America and the Caribbean countries) at $0.072 \%$, over the same period [3]. It is noteworthy that although the Hispanic and Non-Hispanic (English-FrenchDutch-speaking) Caribbean have geographic proximity and share similar climatic conditions [14, 28], Barbados in particular and rest of the non-Hispanic Caribbean, in general, have consistently recorded a significantly higher incidence rate of dengue during the epidemic years as compared with the Hispanic Caribbean [6]. More movement of people among the different English-speaking Caribbean countries with greater potential for different serotypes introduction and reintroduction could be a factor in the higher incidence of dengue in these countries [29]. This is further supported by the fact that the circulating serotypes in the neighboring English-speaking Caribbean are similar to those circulations in Barbados [9]. Higher population density in Barbados compared with the other Caribbean countries may also facilitate greater transmission of dengue in this population. Lastly, this higher incidence rate of dengue in Barbados, as seen in this study, compared with the number of cases reported to the PAHO from Barbados and other Caribbean countries may partly be due to the underreporting. Most of the Caribbean and Latin American countries use national passive surveillance for reporting dengue to the PAHO and under-reporting is a wellrecognised problem in this system. Several studies from the Latin Americas have shown that the number of dengue reported to the PAHO are several folds lower than the actual number of cases [30, 31].

The annual incidence rate of dengue in Barbados, at least for some of the years during the study, was also higher than that reported from some other hyper-endemic countries in the Americas [26, 27]. For the year 2010, Brazil reported an annual incidence rate of $0.5 \%$ and Columbia reported an incidence rate of $0.6 \%$ as compared with our own rate of $0.71 \%$ [26, 27]. A higher number of cases were seen in Barbados, as compared with both, the Americas as a whole and the English-speaking Caribbean, during all of the epidemic years [6]. Whether the higher transmission of dengue, in general, over the study period in this country, is due to the variation in the rainfall pattern in the region, the intensity of vector control measures or due to the higher population density of Barbados, needs further study.

During 2014 and 2015, the pattern of dengue occurrence in Barbados and other English/Dutch/French-speaking Caribbean have reported a lower number and incidence rate of dengue compared with the Americas as a whole [6]. The intensity of DENV transmission through the years - with the circulation of several serotypes, as shown in this study - may have led to the decrease in the number of cases. This decline may also be due to better 


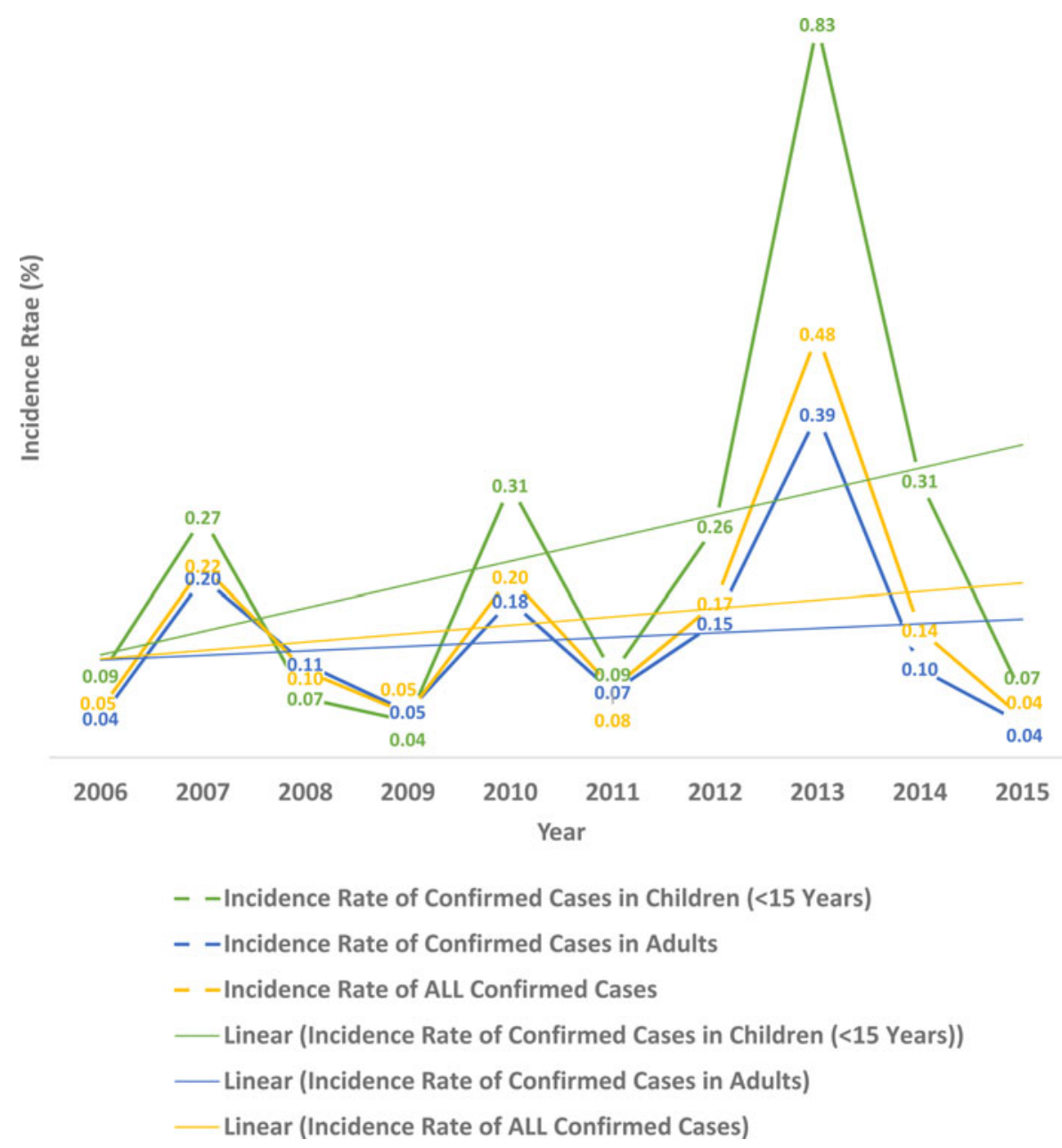

Fig. 4. Incidence rate of confirmed dengue in children and adults, Barbados, 2006-2015. fogging and vector control measures after the massive 2013 epidemics. These dynamics of dengue transmission was further complicated by the introduction and subsequent epidemics of chikungunya in this population during the 2014 and 2015 and its possible effects on dengue transmission given that they both share the same vector [32].

A closer look at the data for the Caribbean region reveals progressively higher peaks and troughs in the number and incidence of dengue over the study years. However, in Barbados and other English-speaking Caribbean, although the peaks have had a progressive rise, the troughs have not shown a similar rise, during the study period (Table 1). This lack of carrying forward of highintensity viral transmission of the epidemic into the following years may be due to the intense fogging and other mosquito control measures triggered during the epidemic and continued for several months thereafter. This can be described as a 'need based mosquito control program'.

Although a higher number of dengue cases were seen during the years with more rainfall, compared with the years with less rainfall (Fig. 1), no significant correlation between the mean annual rainfall and number of cases was seen over the study period. Analysis of monthly cases and weather patterns showed that dengue cases consistently peaked during the rainy months of the year during all of the study years. There was a significant correlation between the mean monthly number of cases and the mean monthly rainfall as well as the relative humidity (Fig. 2). These findings support the facts that the rainfall distribution and density, and its interplay with other weather conditions such as temperature and humidity are important for vector density and dengue transmission than simply the amount of rainfall $[13,14]$. The dengue occurrence and weather patterns suggest a complex relationship rather than a simple one, as also reported in some other more elaborate studies on dengue and climatic factors $[13,14]$. It is likely that the distribution pattern of the rainfall and its interplay with temperature and other weather variables plays an important role in the vector breeding and dengue transmission. In recent studies from Puerto Rico (Spanish-speaking Caribbean) and Guadeloupe from this region, it was shown that the occurence of dengue cases were significantly associated with multiple weather variables $[14,28]$.

Dengue in this population is predominantly a childhood infection with the highest proportion of cases seen in the age group 015 years. Another population-based longitudinal study of dengue, both in children and adults from Philippines, which is also hyperendemic for dengue, has also reported the highest proportion of cases in the age group 0-15 years [19]. However, a similar age distribution pattern has been reported in a systematic literature review of retrospective studies from some other countries in the Latin America [26, 27, 33]. The median age of the patients with confirmed dengue in this population has decreased from 27 years in a previous study of dengue during the 2000-2009 from this country, to 25 years in the present study [8]. A similar shift in the age of dengue has been seen in some other long-term studies from the Americas [27, 34]. In south-east Asian countries, 
where all the serotypes (DENV-1-4) are circulating, DF is typically acknowledged to be a disease of early childhood [34]. This is expected to happen when there is a long lasting transmission of the viruses in one setting; adults have been infected with all four serotypes and this trend pushes infection towards adolescents and children. When dengue is introduced into a naive population, it predominantly affects the adults $[35,36]$. Infection with any one dengue serotype confers lifelong immunity to that serotype and repeated exposure with different serotype over time results in decreasing pool of susceptible adults. Studies from south-east Asia have shown a similar shift in the affected age group pattern $[19,37]$. A previous sero-epidemiologic study in this population and another study from the region showing a high dengue seroprevalence by early adulthood explains the age distribution of symptomatic dengue in this population $[22,38,39]$.

Another notable observation in this study was that of a significantly higher proportion of both the suspected and the confirmed cases of dengue among females which is unlike the high male to female ratios reported in studies from south-east Asia [40, 41]. A male to female ratio similar to ours has been observed in other countries from the Americas $[1,38]$. Aedes aegypti mosquitoes are mostly indoor in their habitat. Culturally, in this population, as in many others, females are more likely to be indoor thereby increasing their chance of mosquito bites and risk of dengue. However, the sex distribution observed in a surveillance study may be reflective of the demography of this country with a higher proportion of females (51.9\%) in the general population [18].

The overall trend in the annual incidence rate of both suspected and the confirmed dengue in this country over the 10 -year study period has been upward. The highest incidence rate of $0.48 \%$ and $1.0 \%$ for the confirmed and suspected dengue respectively was noted during the worst epidemic in 2013. Of note, the incidence rates of both the suspected and confirmed cases of dengue have progressively peaked at higher levels during the epidemic years. Despite the yearly variations and cyclical epidemics, trend analysis of the incidence of dengue in the period 2006-2015 showed an overall increase in incidence over time that was statistically significant. A similar trend has been observed in reports from some other countries in the Americas [26, 27, 35]. Increasing incidence rate was more pronounced and significant among children as compared with adults where a similar increase in incidence was noted but it was statistically not significant. This finding was observed in the absence of any dramatic change in the climatic variables during the study period. As explained earlier, this disproportionate increase in incidence rate among children with shifting of the median age of dengue to younger age is expected from the long-standing transmission in this setting.

The major limitation of this study is that not all persons who have dengue symptoms always see a doctor. Persons with dengue without febrile illness or other symptoms would have been missed in this study. Also, younger children who may present with either nonspecific febrile illness or atypical features such as gastrointestinal symptoms can be diagnosed with other viral syndromes and dengue may not have been suspected. Therefore, the incidence rate of symptomatic confirmed dengue in this study may have been underestimated. Another important limitation is that inadequate data were available on other circulating viruses with clinical presentations similar to dengue especially, Chikungunya and Zika. Febrile patients may be infected by other viruses than dengue; the use of reported cases in a setting where other arboviruses circulate may over-represent the incidence rate of suspected dengue.
In conclusion, there is a high transmission rate of dengue in this country and it is higher than those reported from the Hispanic Caribbean countries. A shorter inter-epidemic period was seen in recent years. There was an inconsistent relationship between the annual rainfall and the dengue occurrence, however, a more consistent relationship was observed between mean monthly rainfall as well as mean monthly humidity percentage and dengue occurrence. These facts point to a complex relationship with weather conditions especially rainfall and humidity and dengue transmission. The most important finding from this study was that the highest incidence of dengue was seen among children. As compared with an earlier reporting period there is a shift in the dengue occurrence to the lower age group. Overall, there was an increasing trend in the incidence of dengue in this population. This increasing trend was more pronounced in the incidence rate among children as compared with the adults.

Acknowledgements. We thank Miss Nicole Clarke-Greenidge at the Dengue public health laboratory for her assistance in data collection. We also thank public health nurse responsible for dengue reporting and surveillance for her assistance with cross-checking of data. We thank Prerna Singh and Pranav Kumar Singh for checking the manuscript for English language.

\section{References}

1. San Martin JL, et al. (2010) The epidemiology of dengue in the Americas over the last three decades: a worrisome reality. American Journal of Tropical Medicine and Hygiene 82, 128-135.

2. Shepard DS, et al. (2011) Economic impact of dengue illness in the Americas. American Journal of Tropical Medicine and Hygiene 84, 200207.

3. Cafferata ML, et al. (2013) Dengue epidemiology and burden of disease in Latin America and the Caribbean: a systematic review of the literature and meta-analysis. Science Direct 2, 347-356.

4. Murray NE, Quam MB and Wilder-Smith A (2013) Epidemiology of dengue: past, present and future prospects. Clinical Epidemiology 5, 299-309.

5. Bhatt S, et al. (2013) The global distribution and burden of dengue. Nature 25, 504-507.

6. Pan American Health Organization (PAHO) Number of reported cases of dengue and dengue hemorrhagic fever (DHF), Region of the Americas (by country and subregion). From 1995 through 2017. Available at http:// www.paho.org/hq/index.php?option=com_topics\&view=readall\&cid=3273\& Itemid=40734 (Accessed 22 December 2017).

7. Chadee DD, Mahabir RS and Sutherland JM (2012) Dengue fever epidemiology and control in the Caribbean: a status report (2012). Caribbean Medical Journal 74(1), 17-21.

8. Kumar A, et al. (2015) Epidemiological trend and clinical observations among children and adults with dengue in Barbados. West Indian Medical Journal 64, 110-116.

9. Campione-Piccardo J, et al. (2003) Dengue viruses in the Caribbean. Twenty years of dengue virus isolates from the Caribbean epidemiology centre. West Indian Medical Journal 52, 191-198.

10. Gittens-St Hilaire $\mathbf{M}$ and Clarke-Greenidge $\mathbf{N}$ (2008) An analysis of the subtypes of dengue fever infections in Barbados 2003-2007 by reverse transcriptase polymerase chain reaction. Virology Journal 5, 152-157.

11. Campbell KM, et al. (2013) The complex relationship between weather and dengue virus transmission in Thailand. American Journal of Tropical Medicine and Hygiene 89, 1066-1080.

12. Thai KTD, et al. (2010) Dengue dynamics in Binh Thuan province, southern Vietnam: periodicity, synchronicity and climate variability. PLoS Neglected Tropical Diseases 4, e747.

13. Stoddard ST, et al. (2014) Long-Term and seasonal dynamics of dengue in Iquitos, Peru. PLoS Neglected Tropical Diseases 8(7), e3003. doi: 10.1371/journal.pntd.0003003. 
14. Méndez-Lázaro $\mathbf{P}$, et al. (2014) Assessing climate variability effects on dengue incidence in San Juan, Puerto Rico. International Journal of Environmental Research and Public Health 11, 9409-9428. doi: 10.3390/ ijerph110909409.

15. Díaz-Quijano FA and Waldman EA (2012) Factors associated with dengue mortality in Latin America and the Caribbean, 1995-2009: an ecological study. American Journal of Tropical Medicine and Hygiene 86, 328-334.

16. Stewart-Ibarra AM and Lowe R (2013) Climate and non-climate drivers of dengue epidemics in Southern Coastal Ecuador. American Journal of Tropical Medicine and Hygiene 88, 971-981.

17. Teixeira MG, et al. (2008) Recent shift in age pattern of dengue hemorrhagic fever, Brazil. Emerging Infectious Diseases 14, 1663-1668.

18. Barbados Statistical Services (2012) 2010 population and housing census. pp. 2-3. Available at http://www.barstats.gov.bb/files/documents/PHC 2010_Census_Volume_1.pdf.

19. Alera MT, et al. (2016) Incidence of dengue virus infection in adults and children in a prospective longitudinal cohort in the Philippines. PLoS Neglected Tropical Diseases 10, e0004337.

20. Kanakaratne N, et al. (2009) Severe dengue epidemics in Sri Lanka, 2003 2006. Emerging Infectious Diseases 15, 192-199.

21. Branco MS, et al. (2015) Dengue in the State of Rio Grande do Norte, Brazil, 2010-2012. Tropical Medicine and International Health 20, 1707-1710.

22. Kumar A and Nielsen AL (2015) Trends in the patterns of IgM and IgG antibodies in febrile persons with suspected dengue in Barbados, an English-speaking Caribbean country, 2006-2013. Journal of Infection and Public Health 8, 583-592.

23. Endy TP, Yoon IK and Mammen MP (2010) Prospective cohort studies of dengue viral transmission and severity of disease. Current Topics in Microbiology and Immunology 338, 1-13.

24. Ramadona AL, et al. (2016) Prediction of dengue outbreaks based on disease surveillance and meteorological data. PLOS ONE 11, e0152688.

25. WHO (2017) Dengue and severe dengue. Available at http://www.who.int/ mediacentre/factsheets/fs117/en/.

26. Teixeira MG, et al. (2013) Epidemiological trends of dengue disease in Brazil (2000-2010): a systematic literature search and analysis. PLoS Neglected Tropical Diseases 7, e2520.

27. Villar LA, et al. (2015) Epidemiological trends of dengue disease in Colombia (2000-2011): a systematic review. PLoS Neglected Tropical Diseases 9, e0003499.
28. Gharbi M, et al. (2011) Time series analysis of dengue incidence in Guadeloupe, French West Indies: forecasting models using climate variables as predictors. BMC Infectious Diseases 11, 166.

29. Vaughn DW (2000) Invited commentary: Dengue lessons from Cuba. American Journal of Epidemiology 152(9), 800-803.

30. Standish K, et al. (2010) High dengue case capture rate in four years of a cohort study in Nicaragua compared to national surveillance data. PLoS Neglected Tropical Diseases 4(3), article, e633. doi: 10.1371/ journal.pntd.0000633.

31. Sarti E, et al. (2016) A comparative study on active and passive epidemiological surveillance for dengue in five countries of Latin America. International Journal of Infectious Diseases 44, 44-49. doi: 10.1016/ j.ijid.2016.01.015.

32. Kumar A, Best C and Benskin G (2016) Epidemiology, clinical and laboratory features and course of chikungunya among a cohort of children during the first Caribbean epidemic. Journal of Tropical Pediatrics 62, 261-267

33. Do TT, et al. (2014) Climatic-driven seasonality of emerging dengue fever in Hanoi, Vietnam. BMC Public Health 14, 1078.

34. Dante's HG, Farfa'n-Ale JA and Sarti E (2014) Epidemiological trends of dengue disease in Mexico (2000-2011): a systematic literature search and analysis. PLoS Neglected Tropical Diseases 8, e3158.

35. Bhatia R, Dash AP and Sunyoto T (2013) Changing epidemiology of dengue in South-East Asia. South-East Asia Journal of Public Health 2, 23-27.

36. Rahman M, et al. (2002) First outbreak of dengue hemorrhagic fever in Bangladesh. Emerging Infectious Diseases 8, 738-740.

37. Olkowski S, et al. (2013) Reduced risk of disease during postsecondary dengue virus infections. Journal of Infectious Diseases 208, 1026-1033.

38. Morrison AC, et al. (2010) Epidemiology of dengue virus in Iquitos, Peru 1999 to 2005: interepidemic and epidemic patterns of transmission. PLoS Neglected Tropical Diseases 4, e670.

39. Dayan G, et al. (2015) Prospective cohort study with active surveillance for fever in four dengue endemic countries in Latin America. American Journal of Tropical Medicine and Hygiene 93, 18-23.

40. Prasith N, et al. (2013) Assessment of gender distribution in dengue surveillance data, the Lao People's Democratic Republic. Western Pacific Surveillance and Response Journal 4(2), 17-24. doi: 10.5365/wpsar.2012. 3.4.020.

41. Anker M and Arima Y (2011) Male-female differences in the number of reported incident dengue fever cases in six Asian countries. Western Pacific Surveillance and Response Journal 2(2), 17-23. doi: 10.5365/ wpsar.2011.2.1.002. 